\title{
Asymptotic Neutrality of Diatomic Molecules
}

\author{
Jan Philip Solovej*** \\ Department of Mathematics, Princeton University, Princeton, NJ 08544, USA
}

\begin{abstract}
We use the no-binding theorem of Thomas-Fermi theory to prove that a large diatomic molecule is "almost" neutral. That is to say, that if the total nuclear charge is $Z$ then the number $N$ of electrons required for the diatomic molecule to be stable satisfies $\lim _{Z \rightarrow \infty} N / Z=1$. In contrast to the atomic case the emphasis here is on the lower bound on $N$. Our analysis will imply a new bound on the size of the molecule. These results are proved in the Born-Oppenheimer approximation. We also give bounds on $N$ which hold for all $Z$ by a very elementary method, not assuming the Born-Oppenheimer approximation.
\end{abstract}

\section{Introduction}

In this paper we will study stability of diatomic molecules. The Hamiltonian for a diatomic molecule is

$$
\begin{aligned}
H_{\mathrm{tot}}= & -\frac{1}{2 M_{1}} \Delta_{\mathbf{R}_{1}}-\frac{1}{2 M_{2}} \Delta_{\mathbf{R}_{2}}-\frac{1}{2 m} \sum_{i=1}^{N} \Delta_{\xi_{i}} \\
& -\sum_{i=1}^{N}\left(\frac{Z_{1}}{\left|\xi_{i}-\mathbf{R}_{1}\right|}+\frac{Z_{2}}{\left|\xi_{i}-\mathbf{R}_{2}\right|}\right)+\sum_{i<j} \frac{1}{\left|\xi_{i}-\xi_{j}\right|}+\frac{Z_{1} Z_{2}}{\left|\mathbf{R}_{1}-\mathbf{R}_{2}\right|} \\
= & -\frac{1}{2\left(M_{1}+M_{2}\right)} \Delta_{\mathbf{R}_{C M}}+H(N, \underline{Z}),
\end{aligned}
$$

where $H(N, \underline{Z})$ is the Hamiltonian with the center of mass motion of the nuclei removed, $\underline{Z}=\left(Z_{1}, Z_{2}\right)$. $\mathbf{R}_{C M}=(\lambda+1)^{-1}\left(\lambda \mathbf{R}_{1}+\mathbf{R}_{2}\right)$ is the center of mass of the nuclei, where $\lambda=M_{1} / M_{2} . \xi_{i}, i=1, \ldots, N$ are electron coordinates. We are not

\footnotetext{
* Work supported by a Danish Research Academy fellowship and U.S. National Science Foundation grant PHY-85-15288-A03

** Adress from September 1989: Department of Mathematics, The University of Michigan, Ann Arbor, Michigan 48109, USA
} 
assuming that $\lambda$ is equal to $Z_{1} / Z_{2}$, but we will assume that $M_{i} / Z_{i}$ is of the order of the proton mass $m_{p}$.

Introducing the coordinates

$$
\mathbf{R}=\left(\mathbf{R}_{1}-\mathbf{R}_{2}\right) \text { and } \quad x_{i}=\xi_{i}-\mathbf{R}_{C M},
$$

we can write

$$
\begin{aligned}
H(N, \underline{Z})= & -\frac{M_{1}+M_{2}}{2 M_{1} M_{2}} \Delta_{\mathbf{R}}-\frac{1}{2 m} \sum_{i} \Delta_{x_{i}}-\frac{1}{2\left(M_{1}+M_{2}\right)}\left(\sum_{i=1}^{N} \nabla_{i}\right)^{2} \\
& -\sum_{i=1}^{N}\left(\frac{Z_{1}}{\left|x_{i}-(\lambda+1)^{-1} \mathbf{R}\right|}+\frac{Z_{2}}{\left|x_{i}+\lambda(\lambda+1)^{-1} \mathbf{R}\right|}\right) \\
& +\sum_{i<j} \frac{1}{\left|x_{i}-x_{j}\right|}+\frac{Z_{1} Z_{2}}{R} .
\end{aligned}
$$

$H(N, \underline{Z})$ is an operator which in the physically relevant case acts on the fermionic space $L^{2}\left(\mathbb{R}_{\mathbf{R}}^{3}\right) \otimes\left(\bigwedge_{i=1}^{N} L^{2}\left(\mathbb{R}_{x_{i}}^{3} ; \mathbb{C}^{2}\right)\right)$, but in general one can consider other spaces with different kinds of symmetry.

The question we are interested in here is when $H(N, \underline{Z})$ has a stable ground state $\psi$, stable meaning with an eigenvalue (energy) $E(N, \underline{Z})$ below the continuum. In [9] it was proved that neutral diatomic molecules are always stable, since there is at least a van der Waals attraction between the atoms (this was proved in the limit where the masses of the nuclei are large compared with the mass of the electron).

On the other hand if a molecule is too far from being neutral it cannot be stable. There are two different cases of instability:

1. It was proved in [7] that if $N>2\left(Z_{1}+Z_{2}\right)+2$ then the molecule will break up into a molecule with fewer electrons and at least one free electron.

2. In a recent paper [11] Ruskai points out that if $N$ is too small (or equivalently $Z_{1}, Z_{2}$ too large) then the molecule breaks up into two independent atoms.

In her paper Ruskai not only proves this, she also derives asymptotic $(N \rightarrow \infty)$ bounds on the critical nuclear charges in the fermionic case. Here we first present an elementary proof in the spirit of Lieb [7] which gives bounds on the critical charges for all $N$ indepedent of particle symmetry. Our main result, however, is that for fermions diatomic molecules must be asymptotically neutral. The asymptotic bound is derived in the Born-Oppenheimer approximation, $M_{1}=$ $M_{2}=\infty$, while the global bound is for completely dynamic nuclei. In the BornOppenheimer approximation $\mathbf{R}$ is a parameter and the energy is a function of $R=|\mathbf{R}|$,

$$
E(N, \underline{Z}, R)=\inf \operatorname{spec} H(N, \underline{Z}, \mathbf{R}),
$$

$H(N, \underline{Z}, \mathbf{R})$ acts on $\left(\bigwedge_{i=1}^{N} L^{2}\left(\mathbb{R}_{x_{i}}^{3} ; \mathbb{C}^{2}\right)\right)$. In this case we define

$$
E(N, \underline{Z})=\inf _{R} E(N, \underline{Z}, R) .
$$


Here having a stable ground state means that the following two conditions are satisfied.

(a) $E(N, \underline{Z})<E(N, \underline{Z}, R=\infty)$.

(b) $E(N, \underline{Z})$ corresponds to a stable ground state of $H\left(N, \underline{Z}, \mathbf{R}_{0}\right)$ for some $\mathbf{R}_{0}$, i.e., $E(N, \underline{Z})$ is an eigenvalue strictly below the continuum for $H\left(N, \underline{Z}, \mathbf{R}_{0}\right)$.

It is this notion of stability which was proved in [9]. It is worth noticing that $E(R=\infty)$ is the sum of the energies of two non-interacting atoms, i.e.,

$$
E(N, \underline{Z}, R=\infty)=\min _{N_{1}+N_{2}=N}\left(E^{\text {atom }}\left(Z_{1}, N_{1}\right)+E^{\text {atom }}\left(Z_{2}, N_{2}\right)\right) .
$$

Thus (a) says that for a molecule to be stable the energy must be smaller than the energy of any pair of corresponding independent atoms. The interpretation of (b) is that even if the molecule does not split into independent atoms we still have to make sure that all the electrons are bounded.

If (a) does not hold we have instability as in case 2 above, and if (b) does not hold we have instability as in case 1 .

Related to the question of neutrality are the questions of what the distance $R(N, \underline{Z})$ between the nuclei is in a stable molecule [i.e., $\left|\mathbf{R}_{0}\right|$ in (b) above], and what the binding energy is for such a molecule. The binding energy is defined as

$$
\Delta E_{b}(N, \underline{Z})=E(N, \underline{Z}, R=\infty)-E(N, \underline{Z}) .
$$

For fermions it is easy to prove [see (21) below] that

$$
R \geq C\left(Z_{1}+Z_{2}\right)^{-1 / 3} .
$$

The binding energy is of course smaller than the absolute value of the total energy, but to the best of my knowledge it is not easy to get a better bound. For fermions it is possible to improve both of these results.

Theorem 1. If $H(N, \underline{Z})$ has a stable ground state then, independent of particle symmetry,

$$
(2 / 3)(1+a)^{-1} \frac{Z_{1} Z_{2}}{Z_{1}+Z_{2}} \leq N,
$$

where a given in (23) below is the effect of the finite nuclear mass, it is of order $\mathrm{m} / \mathrm{m}_{p}$. If $M_{1}, M_{2}=\infty$, then

$$
\text { (2/3) } \frac{Z_{1} Z_{2}}{Z_{1}+Z_{2}} \leq N<2\left(Z_{1}+Z_{2}\right)+2 \text {. }
$$

The upper bound in (4) is Lieb's result ${ }^{1}$. The lower bound will be proved in Sect. 3 below.

The asymptotic neutrality is formulated in

\footnotetext{
${ }^{1}$ For dynamic nuclei Lieb only had a weaker result, see [7] Sect. VI(B). In [10] Ruskai gives an upper bound to $N$ for completely dynamic nuclei
} 
Theorem 2 (Asymptotic Neutrality). Given $z_{1}, z_{2}>0$, with $z_{1}+z_{2}=1$. Let $\left(Z_{1}, Z_{2}\right)=\left(Z z_{1}, Z z_{2}\right)$. If $M_{1}, M_{2}=\infty$ and $H(N, \underline{Z})$ has a stable ground state on the fermionic space, then

$$
\lim _{Z \rightarrow \infty} \frac{N}{Z}=1 \quad \text { and } \quad \lim _{Z \rightarrow \infty} \frac{\Delta E_{b}(N, \underline{Z})}{E(N, \underline{Z})}=0 .
$$

In fact there exist $C_{1}, C_{2}, C_{3}, \varepsilon>0$ depending only on $z_{1}, z_{2}$ such that

$$
\begin{aligned}
|Z-N| & <C_{1} Z^{1-\varepsilon}, \\
\Delta E_{b}(N, \underline{Z}) & <C_{2} Z^{(7 / 3)(1-\varepsilon)}, \\
R(N, \underline{Z}) & >C_{3} Z^{-(1 / 3)(1-\varepsilon)} .
\end{aligned}
$$

Remark. Intuitively it might be thought that Theorem 2 is a simple consequence of electrostatics. But as we will now show the theorem would be wrong without the assumption of fermions. The corresponding non-neutrality for "bosonic" atoms was shown in [2]. Since the method used there essentially applies for molecules as well, we will only sketch the proof here and refer to [2] for details. We consider a molecule with $Z_{1}=Z_{2}=Z / 2$, without particle symmetry. We will show that stability condition (a) will be satisfied as long as $N / Z$ remains in some open interval around 1 . That (b) is also satisfied can be proved exactly as it was done for atoms in [2]. As in [2] an easy variational argument gives the following upper bound to the molecular energy

$$
E(N, Z, R) \leq-Z^{3} e(N / Z, R Z)
$$

where $-e$ is the Hartree energy

$$
e(n, r)=-\inf \left\{L(\varrho, \underline{\mathbf{r}}) \mid \int \varrho=n\right\}, \quad \underline{\mathbf{r}}=\left(\mathbf{r}_{1}, \mathbf{r}_{2}\right) ; r=|\underline{\mathbf{r}}|,
$$

and $L$ is the functional

$$
L(\varrho, \underline{\mathbf{r}})=\int(\nabla \sqrt{\varrho})^{2} d x-\frac{1}{2} \int\left(\left|x-\mathbf{r}_{1}\right|^{-1}+\left|x-\mathbf{r}_{2}\right|^{-1}\right) \varrho d x+\frac{1}{2} \int \frac{\varrho(x) \varrho(y)}{|x-y|} d x d y .
$$

On the other hand for atoms it was proved in [2] that

$$
E^{\text {atom }}\left(N_{1}, Z_{1}\right) \geq-Z^{3}\left[e^{\text {atom }}\left(N_{1} / Z_{1}, Z_{1} / Z\right)^{1 / 2}+b Z^{-2 / 3}(N / Z)^{5 / 6}\right]^{2},
$$

where $b>0$ is a constant. It was proved in [6] (in the more general setting of the TFW-model) that for $n=1$ there exists $r_{0}<\infty$ such that

$$
\begin{aligned}
& -e\left(n=1, r_{0}\right)<\min _{n_{1}+n_{2}=1}\left[-e^{\text {atom }}\left(n_{1}, z_{1}=\frac{1}{2}\right)-e^{\text {atom }}\left(n_{2}, z_{2}=\frac{1}{2}\right)\right] \\
& =-2 e^{\text {atom }}(n=1 / 2,1 / 2) .
\end{aligned}
$$

From continuity we can thus find $n_{c}^{-}<1<n_{c}^{+}$such that (9) holds for all $n_{c}^{-}<n<n_{c}^{+}$. But then it is easy to see that if $n_{c}^{-}<N / Z<n_{c}^{+}, R=r_{0} Z^{-1}$ and 
$Z$ is sufficiently large then

$$
\begin{aligned}
E(N, Z, R) \leq & -Z^{3} e\left(N / Z, r_{0}\right)<-Z^{3} \min _{N_{1}+N_{2}=N} \\
& \times\left\{\left[e^{\text {atom }}\left(N_{1} / Z\right)^{1 / 2}+b Z^{-2 / 3}\left(N_{1} / Z\right)^{5 / 6}\right]^{2}\right. \\
& \left.+\left[e^{\text {atom }}\left(N_{2} / Z\right)^{1 / 2}+b Z^{-2 / 3}\left(N_{2} / Z\right)^{5 / 6}\right]^{2}\right\} \\
\leq & \min _{N_{1}+N_{2}=N}\left(E^{\text {atom }}\left(N_{1}, Z_{1}\right)+E^{\text {atom }}\left(N_{2}, Z_{2}\right)\right) .
\end{aligned}
$$

It is therefore clear that (a) above is satisfied as long as $n_{c}^{-}<N / Z<n_{c}^{+}$and $Z$ is large enough. This together with the fact that (b) also holds shows that Theorem 2 is not valid without the assumption of fermionic symmetry.

We prove Theorem 2 by comparing with Thomas-Fermi (TF) theory, in contrast to the above remark where we used the Hartree model. Indeed TF theory is known to approximate the true quantum energy of fermionic atoms and molecules to leading order.

The crucial fact about TF theory that will be needed here is that molecules do not bind in this model. This is what is known as Teller's no-binding Theorem. While this is usually considered a fault of the model, in fact, it just means that the binding energy is of lower order than the full energy, i.e., (7) above.

The actual repulsion between atoms in the TF model was studied in $[1,3]$. Their results will allow us to conclude (8). This comparison with TF theory is carried out in Sect. 4.

From (8) we see that $R$ is larger than the TF scale $Z^{-1 / 3}$. The TF density of the molecule is therefore almost a sum of two atomic densities. We show in Sect. 5 exactly how this approximation depends on $R$. This is of importance in proving the bound (6).

Equation (6) consists of an upper and a lower bound to $N$. The upper bound, which is very similar to upper bounds in the atomic case (see $[8,5$, or 12]), is proved in Sect. 8. The lower bound is special for molecules and rely heavily on (8). This is presented in Sect. 7.

Both the upper and the lower bound are proved by using TF theory to control the true ground state density

$$
\varrho(x)=N \sum_{\sigma} \int\left|\psi\left(x, \sigma ; x_{2}, \sigma_{2} ; \ldots ; x_{N}, \sigma_{N}\right)\right|^{2} d\left(x_{2}, \sigma_{2}\right) \ldots d\left(x_{N}, \sigma_{N}\right),
$$

( $x$ is a space variable, $\sigma$ is a spin variable) and 2-point correlation

$$
\varrho^{(2)}(x, y)=N(N-1) \sum_{\sigma_{1}, \sigma_{2}} \int\left|\psi\left(x, \sigma_{1} ; y, \sigma_{2} ; \ldots ; x_{N}, \sigma_{N}\right)\right|^{2} d\left(x_{3}, \sigma_{3}\right) \ldots d\left(x_{N}, \sigma_{N}\right) .
$$

These quantities are estimated in Sect. 6 .

The lower bound on $N$ is proved by showing that this bound is necessary for the stability condition (a) to be satisfied. While the upper bound is proved by showing it is necessary if (b) is also to hold.

In Sect. 2 we begin by describing a general localization argument which will be used in Sect. 3 to prove the global lower bound on $N$ and in Sect. 7 to prove the asymptotic lower bound. 


\section{Localization Argument}

We will consider two-cluster decompositions $\alpha=\left(\alpha_{1}, \alpha_{2}\right)$ of $\{1, \ldots, N\}$. The intercluster potential is

$$
I_{\alpha}=-\sum_{i \in \alpha_{2}} \frac{Z_{1}}{\left|x_{i}-(\lambda+1)^{-1} \mathbf{R}\right|}-\sum_{i \in \alpha_{1}} \frac{Z_{2}}{\left|x_{i}+\lambda(\lambda+1)^{-1} \mathbf{R}\right|}+\sum_{\substack{i \in \alpha_{1} \\ j \in \alpha_{2}}} \frac{1}{\left|x_{i}-x_{j}\right|}+\frac{Z_{1} Z_{2}}{R} .
$$

The cluster Hamiltonian is $H_{\alpha}=H-I_{\alpha} . H_{\alpha}$ is an operator on the space of functions satisfying the appropriate symmetry condition in the two clusters separately. By a change of coordinates of the form

$$
\mathbf{R}^{\prime}=\mathbf{R}+t_{1} \sum_{i \in \alpha_{1}} x_{i}+t_{2} \sum_{i \in \alpha_{2}} x_{i}
$$

and

$$
y_{i}=\left\{\begin{array}{rr}
x_{i}-(\lambda+1)^{-1} \mathbf{R}, & i \in \alpha_{1} \\
x_{i}+\lambda(\lambda+1)^{-1} \mathbf{R}, & i \in \alpha_{2}
\end{array}\right.
$$

for some constants $t_{1}, t_{2}>0$, it is easy to see that $H_{\alpha}=-(2 \tilde{M}) \Delta_{\mathbf{R}^{\prime}}+H_{\alpha_{1}}+H_{\alpha_{2}}$. Here $\tilde{M}$ is a certain reduced mass and $H_{\alpha_{k}}$ is the atomic Hamiltonian

$$
H_{\alpha_{k}}=\sum_{i \in \alpha_{k}}\left(-\frac{1}{2 m} \Delta_{y_{i}}-\frac{Z_{k}}{\left|y_{i}\right|}\right)-\frac{1}{2 M_{k}}\left(\sum_{i \in \alpha_{k}} \nabla_{y_{i}}\right)^{2}+\sum_{i<j} \frac{1}{\left|y_{i}-y_{j}\right|} .
$$

By the Weyl criterion we easily conclude that if $H$ has a stable ground state then

$$
E=\inf \operatorname{spec} H \leq E_{\alpha}=\inf \operatorname{spec} H_{\alpha} .
$$

The first step in the localization argument is to choose two functions $\chi_{1}$, $\chi_{2} \in H^{1}\left(\mathbb{R}^{3} \times \mathbb{R}^{3}\right)$ with

$$
\chi_{1}(x, \mathbf{R})^{2}+\chi_{2}(x, \mathbf{R})^{2}=1 .
$$

From the simple integration by parts argument used in proving the IMS-formula (see [4]) we get

$$
E\langle\psi|R| \psi\rangle=\left\langle\psi\left|R^{1 / 2} H R^{1 / 2}\right| \psi\right\rangle-\frac{M_{1}+M_{2}}{2 M_{1} M_{2}}\left\langle\psi\left|\frac{1}{4} R^{-1}\right| \psi\right\rangle,
$$

and

$$
\begin{aligned}
\left\langle\psi\left|R^{1 / 2} H R^{1 / 2}\right| \psi\right\rangle & =\left\langle\psi\left|R^{1 / 2} \prod_{i=1}^{N}\left(\chi_{1}\left(x_{i}, \mathbf{R}\right)^{2}+\chi_{2}\left(x_{i}, \mathbf{R}\right)^{2}\right) H R^{1 / 2}\right| \psi\right\rangle \\
& =\sum_{\alpha}\left\langle\psi\left|R^{1 / 2} \chi_{\alpha}^{2} H R^{1 / 2}\right| \psi\right\rangle,
\end{aligned}
$$

where $\chi_{\alpha}(x, \mathbf{R})=\prod_{i \in \alpha_{1}} \chi_{1}\left(x_{i}, \mathbf{R}\right) \prod_{j \in \alpha_{2}} \chi_{2}\left(x_{j}, \mathbf{R}\right)$. Using (11) and the IMS-formula again we obtain

$$
\begin{aligned}
\left\langle\psi\left|R^{1 / 2} H R^{1 / 2}\right| \psi\right\rangle & \geq \sum_{\alpha}\left\{E_{\alpha}\left\langle\psi\left|R \chi_{\alpha}^{2}\right| \psi\right\rangle+\left\langle\psi\left|I_{\alpha} R \chi_{\alpha}^{2}\right| \psi\right\rangle-\left\langle\psi\left|L_{\alpha} R\right| \psi\right\rangle\right\} \\
& \geq \inf _{\alpha} E_{\alpha}\langle\psi|R| \psi\rangle+\sum_{\alpha}\left\{\left\langle\psi\left|I_{\alpha} R \chi_{\alpha}^{2}\right| \psi\right\rangle-\left\langle\psi\left|L_{\alpha} R\right| \psi\right\rangle\right\},
\end{aligned}
$$


where the localization error is

$$
\begin{aligned}
L_{\alpha} & =\frac{M_{1}+M_{2}}{2 M_{1} M_{2}}\left(\nabla_{\mathbf{R}} \chi_{\alpha}\right)^{2}+\frac{1}{2 m} \sum_{i}\left|\nabla_{i} \chi_{\alpha}\right|^{2}+\frac{1}{2\left(M_{1}+M_{2}\right)}\left|\sum_{i} \nabla_{i} \chi_{\alpha}\right|^{2} \\
& \leq \frac{M_{1}+M_{2}}{2 M_{1} M_{2}}\left(\nabla_{\mathbf{R}} \chi_{\alpha}\right)^{2}+\left(\frac{1}{2 m}+\frac{N}{2\left(M_{1}+M_{2}\right)}\right) \sum_{i} R\left|\nabla_{i} \chi_{\alpha}\right|^{2} .
\end{aligned}
$$

The last inequality follows from the Cauchy-Schwartz inequality. From this we easily obtain the final estimate, writing $L$ for the sum of the error term in (12) and all the $L_{\alpha}$,

$$
\begin{aligned}
-\Delta E_{b}(N, \underline{Z})\langle\psi|R| \psi\rangle \geq-\langle\psi|L| \psi\rangle \\
\quad-\sum_{i=1}^{N}\left\langle\psi\left|R \frac{Z_{1}}{\left|x_{i}-(\lambda+1)^{-1} \mathbf{R}\right|} \chi_{2}\left(x_{i}\right)^{2}+R \frac{Z_{2}}{\left|x_{i}+\lambda(\lambda+1)^{-1} \mathbf{R}\right|} \chi_{1}\left(x_{i}\right)^{2}\right| \psi\right\rangle \\
\quad+\sum_{i<j}\left\langle\psi\left|\frac{1}{\left|x_{i}-x_{j}\right|} R\left(\chi_{1}\left(x_{i}\right)^{2} \chi_{2}\left(x_{j}\right)^{2}+\chi_{1}\left(x_{j}\right)^{2} \chi_{2}\left(x_{i}\right)^{2}\right)\right| \psi\right\rangle+Z_{1} Z_{2} .
\end{aligned}
$$

For simplicity we are not displaying the R-dependence of $\chi_{k}$. Using the CauchySchwartz inequality once more we can easily estimate the error by

$$
\begin{aligned}
L \leq & \left.\left.\frac{M_{1}+M_{2}}{8 M_{1} M_{2}} R^{-1}+R N \frac{M_{1}+M_{2}}{2 M_{1} M_{2}} \sum_{i=1}^{N}\left(\nabla_{\mathbf{R}} \chi_{1}\left(x_{i}\right)\right)^{2}+\nabla_{\mathbf{R}} \chi_{1}\left(x_{i}\right)\right)^{2}\right) \\
& +R\left(\frac{1}{2 m}+\frac{N}{2\left(M_{1}+M_{2}\right)}\right) \sum_{i=1}^{N}\left(\left(\nabla_{x} \chi_{1}\left(x_{i}\right)\right)^{2}+\left(\nabla_{x} \chi_{2}\left(x_{i}\right)\right)^{2}\right) .
\end{aligned}
$$

\section{Global Bounds on $N$}

We will now make an explicit choice of localizing functions. These functions will depend on the parameter $\mu=Z_{2} / Z_{1}$. Writing

$$
\bar{x}=x+\frac{\lambda-\mu}{(\mu+1)(\lambda+1)} \mathbf{R} \text { and } \overline{\mathbf{R}}=\frac{1}{\mu+1} \mathbf{R}
$$

we have

$$
\bar{x}+\mu \overline{\mathbf{R}}=x+\lambda(\lambda+1)^{-1} \mathbf{R} \text { and } \bar{x}-\overline{\mathbf{R}}=x-(\lambda+1)^{-1} \mathbf{R} .
$$

Define

$$
\chi_{1}(x)=\frac{|\bar{x}+\mu \overline{\mathbf{R}}|}{\sqrt{(\mu+1)}\left(|\bar{x}|^{2}+\mu \bar{R}^{2}\right)^{1 / 2}} \quad \text { and } \quad \chi_{2}(x)=\frac{\sqrt{\mu}|\bar{x}-\overline{\mathbf{R}}|}{\sqrt{(\mu+1)}\left(|\bar{x}|^{2}+\mu \bar{R}^{2}\right)^{1 / 2}} .
$$

We will assume that $Z_{1} \geq Z_{2}$, i.e., $\mu \leq 1$, the effect of this is to make $\chi_{1}$ greater than $\chi_{2}$ in a larger region of space. Notice that if $\mu=0$ then $\chi_{1}=1$ while $\chi_{2}=0$. It is easy to see that

$$
\chi_{1}^{2}+\chi_{2}^{2}=1
$$


We consider the different terms in (16). We begin with L. A straightforward calculation gives

$$
\begin{aligned}
R\left(\left(\nabla_{x} \chi_{1}(x, \mathbf{R})\right)^{2}+\left(\nabla_{x} \chi_{2}(x, \mathbf{R})\right)^{2}\right) & =\mu(\mu+1)^{-2} R^{3}\left(|\bar{x}|^{2}+\mu \bar{R}^{2}\right)^{-2} \\
& \leq \frac{(\mu+1)^{2}}{\mu} R^{-1}
\end{aligned}
$$

For the $\mathbf{R}$ derivative we first notice that

$$
\begin{aligned}
R\left(\left(\nabla_{\mathbf{R}} \chi_{1}(x, \mathbf{R})\right)^{2}+\left(\nabla_{\mathbf{R}} \chi_{2}(x, \mathbf{R})\right)^{2}\right) & =\chi_{2}^{-2} R\left(\nabla_{\mathbf{R}} \chi_{1}(x, \mathbf{R})\right)^{2} \\
& =\chi_{2}^{-2} R\left(\frac{\lambda-\mu}{(\mu+1)(\lambda+1)} \nabla_{\bar{x}} \chi_{1}+\frac{1}{\mu+1} \nabla_{\overline{\mathbf{R}}} \chi_{1}\right)^{2}
\end{aligned}
$$

Thus

$$
\begin{aligned}
R & \left(\nabla_{\mathbf{R}} \chi_{1}(x, \mathbf{R})^{2}+\left(\nabla_{\mathbf{R}} \chi_{2}(x, \mathbf{R})^{2}\right)\right. \\
& \leq(\mu+1)^{-2} \chi_{2}^{-2} R\left(1+\left(\frac{\lambda-\mu}{\lambda+1}\right)^{2} \mu^{-1}\right)\left(\mu\left(\nabla_{\bar{x}} \chi_{1}\right)^{2}+\left(\nabla_{\overline{\mathbf{R}}} \chi_{1}\right)^{2}\right) \\
& =R \frac{\mu}{(\mu+1)^{2}}\left(1+\left(\frac{\lambda-\mu}{\lambda+1}\right)^{2} \mu^{-1}\right)\left(|\bar{x}|^{2}+\mu \bar{R}^{2}\right)^{-1} \\
& \leq R^{-1}\left(1+\left(\frac{\lambda-\mu}{\lambda+1}\right)^{2} \mu^{-1}\right) .
\end{aligned}
$$

It is now clear that in order to estimate the error term in (16) we must estimate $\left\langle\psi\left|R^{-1}\right| \psi\right\rangle$. We do this by comparing the energy $E$ with the energy of the cluster decomposition corresponding to all electrons in $\alpha_{1}$, i.e.,

$$
E^{\text {atom }}\left(Z_{1}, N\right) \geq E^{\text {atom }}\left(Z_{1}+Z_{2}, N\right)+\left\langle\psi\left|\frac{Z_{1} Z_{2}}{R}\right| \psi\right\rangle .
$$

The last inequality is very easy to derive (see [13]). It was proved in Ruskai [11] using a simple concavity argument that

$$
E^{\text {atom }}\left(Z_{1}+Z_{2}, N\right)-E^{\text {atom }}\left(Z_{1}, N\right) \geq-3 m \sigma_{N} Z_{1} Z_{2},
$$

where

$$
\sigma_{N}= \begin{cases}\frac{1}{2} N & \text { without particle symmetry } \\ \sigma N^{1 / 3} & \text { for fermions }\end{cases}
$$

( $\sigma$ is a constant). Thus

$$
\left\langle\psi\left|R^{-1}\right| \psi\right\rangle \leq 3 m \sigma_{N}
$$

Putting together (17), (19), (20) and (21) we arrive at the following estimate on $L$ independent of particle symmetry

$$
\langle\psi|L| \psi\rangle \leq \frac{3}{2} \frac{(\mu+1)^{2}}{\mu} \sigma_{N} N(1+4 a)=\frac{3}{4} \frac{(\mu+1)^{2}}{\mu} N^{2}(1+4 a),
$$


where

$$
\begin{aligned}
a= & \frac{m}{4}\left(\frac{Z_{1}+Z_{2}}{M_{1}+M_{2}}+m \frac{M_{1}+M_{2}}{M_{1} M_{2}} \frac{Z_{1} Z_{2}}{Z_{1}+Z_{2}}\left[1+\frac{1}{4\left(Z_{1}+Z_{2}\right)}\right]\right. \\
& \left.+m \frac{\left(M_{1} Z_{1}-M_{2} Z_{2}\right)^{2}}{M_{1} M_{2}\left(M_{1}+M_{2}\right)\left(Z_{1}+Z_{2}\right)}\right),
\end{aligned}
$$

here we have inserted the values $\lambda=M_{1} / M_{2}$ and $\mu=Z_{2} / Z_{1}$ and used that we can assume $1 \leq N \leq Z_{1}+Z_{2}$. It is clear that $a$ is of order $O\left(m / m_{p}\right)$.

Returning to (16) it is clear that the term coming from the repulsion between the electrons in different clusters will improve the bound on the critical charges. Unfortunately it is not easy to estimate this term. When proving the asymptotic neutrality for fermions we of course have to use this term, but here we simply neglect it. From (16) we get

$$
0 \geq-\frac{3}{4} \frac{(\mu+1)^{2}}{\mu} N^{2}(1+4 a)-\sum_{i=1}^{N}\left\langle\psi\left|\frac{Z_{2}\left|\bar{x}_{i}+\mu \overline{\mathbf{R}}\right|+\mu Z_{1}\left|\bar{x}_{i}-\overline{\mathbf{R}}\right|}{(\mu+1)\left(\left|\bar{x}_{i}\right|^{2}+\bar{R}^{2}\right)} R\right| \psi\right\rangle+Z_{1} Z_{2} .
$$

We estimate

$$
\begin{aligned}
\left(Z_{2}|\bar{x}+\mu \bar{R}|+\mu Z_{1}|\bar{x}-\overline{\mathbf{R}}|\right)^{2}= & Z_{2}^{2}|\bar{x}+\mu \overline{\mathbf{R}}|^{2}+\mu^{2} Z_{1}^{2}|\bar{x}-\overline{\mathbf{R}}|^{2} \\
& +2 \mu Z_{1} Z_{2}|\bar{x}+\mu \overline{\mathbf{R}}||\bar{x}-\overline{\mathbf{R}}| \\
\leq & \left(Z_{2}^{2}+Z_{1}^{2} \mu\right)\left(|\bar{x}+\mu \overline{\mathbf{R}}|^{2}+\mu|\bar{x}-\overline{\mathbf{R}}|^{2}\right) \\
= & \left(Z_{2}^{2}+Z_{1}^{2} \mu\right)(\mu+1)\left(|\bar{x}|^{2}+\mu \bar{R}^{2}\right) .
\end{aligned}
$$

With the choice $\mu=Z_{2} / Z_{1}$ we obtain

$$
0 \geq-\frac{3}{4} N^{2}(1+4 a)-N \frac{Z_{1} Z_{2}}{Z_{1}+Z_{2}}+\left(\frac{Z_{1} Z_{2}}{Z_{1}+Z_{2}}\right)^{2}
$$

which implies

$$
\frac{Z_{1} Z_{2}}{\left(Z_{1}+Z_{2}\right)} \leq(3 / 2)(1+a) N
$$

This completes the proof of theorem 1:

Remark. If $Z_{1}=Z_{2}$ it is easy to improve (21) so that neglecting the term of order $m / m_{p}$

$$
Z_{1} \leq(1+\sqrt{11 / 3}) N<3 N
$$

Remark. Even though (26) is true for all $Z_{1}$ and $Z_{2}$ we point out that it is trivially satisfied whenever $Z_{2}<N$. As in Ruskai [11] our method does not give any information if $Z_{2}<N$.

\section{Comparison with Thomas-Fermi Theory}

We turn now to the more detailed study of the fermionic case. We assume here that $M_{1}=M_{2}=\infty$, i.e., the Born-Oppenheimer approximation. For simplicity we set $m=1 / 2$ and $\mathbf{R}_{C M}=0$ such that $x_{i}=\xi_{i}$. 
The first step is to compare with TF theory. The TF functional for a diatomic molecule is (for a review of TF-theory see [6])

$$
\begin{aligned}
\mathscr{E}(\varrho)= & \gamma \int \varrho^{5 / 3} d x-Z_{1} \int \varrho(x)\left|x-\mathbf{R}_{1}\right|^{-1} d x-Z_{2} \int \varrho(x)\left|x-\mathbf{R}_{2}\right|^{-1} d x \\
& +D(\varrho, \varrho)+Z_{1} Z_{2} / R
\end{aligned}
$$

where

$$
D(f, g)=(1 / 2) \int f(x)|x-y|^{-1} g(y) d x d y
$$

The physically correct value of $\gamma$ is $\gamma_{p}=\left(3 \pi^{2}\right)^{2 / 3}$ for spin $\frac{1}{2}$ particles. For $N \leq Z$, $\mathscr{E}$ has a unique minimizer with $\int \varrho=N$ that we denote

$$
\varrho_{\mathrm{TF}}^{(\gamma)}(x ; \underline{Z} ; \underline{\mathbf{R}} ; N),
$$

where $\underline{\mathbf{R}}=\left(\mathbf{R}_{1}, \mathbf{R}_{2}\right)$. The neutral density $\varrho_{T F}^{(\gamma)}(x ; \underline{Z} ; \underline{\mathbf{R}} ; Z)$ is the total minimizer for $\mathscr{E}$.

Thomas-Fermi theory has the following scaling property which is crucial to our analysis:

$$
\varrho_{\mathrm{TF}}^{(\gamma)}(x ; \underline{Z} ; \underline{\mathbf{R}} ; N)=\gamma^{-3} Z^{2} \varrho_{\mathrm{TF}}^{(1)}\left(Z^{1 / 3} \gamma^{-1} x ; \underline{z} ; \underline{\mathbf{r}} ; n\right),
$$

where $Z=Z_{1}+Z_{2}, \underline{z}=Z^{-1} \underline{Z}, \underline{\mathbf{r}}=Z^{1 / 3} \gamma^{-1} \underline{\mathbf{R}}$, and $n=Z^{-1} N$. The energy (i.e., the minimum of $\mathscr{E}$ ) scales like

$$
E_{\mathrm{TF}}^{(\gamma)}(\underline{Z} ; R ; N)=\gamma^{-1} Z^{7 / 3} E_{\mathrm{TF}}^{(1)}(\underline{z} ; r ; n)
$$

where $r=\left|\mathbf{r}_{1}-\mathbf{r}_{2}\right|=Z^{1 / 3} \gamma^{-1} R$.

The important fact about $\mathrm{TF}$ theory is that $E_{\mathrm{TF}}$ approximates the true quantum energy to leading order in $Z$. Not only this result but details from its proof will be of importance to us. We therefore give a very short sketch of the proof (see [6]).

Choose $g_{1} \in C_{0}^{\infty}\left(\mathbb{R}^{3}\right)$ radially symmetric, positive and with $\int g_{1}^{2}=1$, and such that $g_{1}(x)=0$ for $|x|>1$. Let $g_{s}(x)=s^{-3 / 2} g_{1}(x / s)$, then $\int g_{s}^{2}=1$. Using the positivity and harmonicity of the kernel $|x-y|^{-1}$ we find for any $\tilde{\varrho} \in L^{1}\left(\mathbb{R}^{3}\right)$,

$$
\begin{aligned}
\sum_{1 \leq i<j \leq N}\left|x_{i}-x_{j}\right|^{-1} & \geq D\left(\sum_{i=1}^{N} g_{s}\left(\cdot-x_{i}\right)^{2}, \sum_{i=1}^{N} g_{s}\left(\cdot-x_{i}\right)^{2}\right)-N D\left(g_{s}^{2}, g_{s}^{2}\right) \\
& =D\left(\sum_{i=1}^{N} g_{s}\left(\cdot-x_{i}\right)^{2}-\tilde{\varrho}, \sum_{i=1}^{N} g_{s}\left(\cdot-x_{i}\right)^{2}-\tilde{\varrho}\right) \\
& +2 D\left(\sum_{i=1}^{N} g_{s}\left(\cdot-x_{i}\right)^{2}, \tilde{\varrho}\right)-D(\tilde{\varrho}, \varrho)-c N s^{-1} .
\end{aligned}
$$

Denoting

$$
K_{N}\left(x_{1}, \ldots, x_{N} ; \tilde{\varrho}\right)=D\left(\sum g_{s}\left(\cdot-x_{i}\right)^{2}-\tilde{\varrho}, \sum g_{s}\left(\cdot-x_{i}\right)^{2}-\tilde{\varrho}\right),
$$


we get the following operator inequality for the diatomic molecule Hamiltonian:

$$
\begin{aligned}
H(N, \underline{Z}, \underline{\mathbf{R}}) \geq & \sum_{i=1}^{N} h_{i}^{(g)}-D(\tilde{\varrho}, \tilde{\varrho})+Z_{1} Z_{2} / R+\sum_{i=1}^{N} h_{i}^{\text {(error) }} \\
& +K\left(x_{1}, \ldots, x_{n} ; \tilde{\varrho}\right)-c N s^{-1},
\end{aligned}
$$

where $h_{i}^{(g)}$ and $h_{i}^{(\text {error) }}$ are one-particle operators defined by ( $\delta$ is a parameter to be chosen later)

$$
h_{i}^{(g)}=-(1-\delta) \Delta_{i}-Z_{1} g_{s}^{2} *\left|x_{i}-\mathbf{R}_{1}\right|^{-1}-Z_{2} g_{s}^{2} *\left|x_{i}-\mathbf{R}_{2}\right|^{-1}+g_{s}^{2} * \tilde{\varrho} *\left|x_{i}\right|^{-1}
$$

and

$$
\begin{aligned}
h_{i}^{\text {(error) }}= & -\delta \Delta_{i}+Z_{1}\left(g_{s}^{2} *\left|x_{i}-\mathbf{R}_{1}\right|^{-1}-\left|x_{i}-\mathbf{R}_{1}\right|^{-1}\right) \\
& +Z_{2}\left(g_{s}^{2} *\left|x_{i}-\mathbf{R}_{2}\right|^{-1}-\left|x_{i}-\mathbf{R}_{2}\right|^{-1}\right) .
\end{aligned}
$$

Choosing $\varrho$ to be the Thomas-Fermi density corresponding to $\gamma=(1-\delta) \gamma_{p}$, i.e.,

$$
\tilde{\varrho}_{N}(x)=\varrho_{\mathrm{TF}}^{\left((1-\delta) y_{p}\right)}(x ; \underline{Z} ; \underline{\mathbf{R}} ; N)
$$

we find, following the coherent state proof in [6] that

$$
\begin{aligned}
H(N, \underline{Z}, \underline{\mathbf{R}}) \geq & E_{\mathrm{TF}}^{\left((1-\delta) \gamma_{p}\right)}(\underline{Z} ; R ; N)-N \int\left|\nabla g_{s}\right|^{2} d x+K\left(x_{1} \ldots, x_{N} ; \tilde{\varrho}\right) \\
& -C\left(\delta^{-3 / 2} Z^{5 / 2} s^{1 / 2}+N s^{-1}\right) \\
\geq & (1-\delta)^{-1} E_{\mathrm{TF}}^{\gamma_{p}}(\underline{Z} ; R ; N)+K\left(x_{1} \ldots, x_{N} ; \tilde{\varrho}\right) \\
& -C\left(\delta^{-3 / 2} Z^{5 / 2} s^{1 / 2}+N s^{-1}+N s^{-2}\right)
\end{aligned}
$$

With the choices $\delta=Z^{-1 / 30}$ and $s=Z^{-1 / 2}$ this becomes

$$
H(N, \underline{Z}, \underline{\mathbf{R}}) \geq E_{\mathrm{TF}}^{\left(\gamma_{p}\right)}(\underline{Z} ; R ; N)+K\left(x_{1} \ldots, x_{N} ; \tilde{\varrho}\right)-C Z^{(7 / 3)(1-\varepsilon)}
$$

with $\varepsilon=1 / 70$. The constant $C$ depends only on $z_{1}$ and $z_{2}$ (not on $n$ since from Theorem $\left.1(2 / 3) z_{1} z_{2} \leq n\right)$.

The famous no-binding Theorem of Teller (see [6]) states that

$$
\Gamma(\underline{Z} ; R ; N) \equiv E_{\mathrm{TF}}(\underline{Z} ; R ; N)-\lim _{R \rightarrow \infty} E_{\mathrm{TF}}(\underline{Z} ; R ; N)>0 .
$$

As $R$ tends to infinity the energy of the molecule will tend to the energy of two independent atoms. i.e., there exist $n_{1}, n_{2}$ with $n_{1}+n_{2}=n$ such that

$$
\lim _{R \rightarrow \infty} E_{\mathrm{TF}}(\underline{Z} ; R ; N)=E_{\mathrm{TF}}^{\text {atom }}\left(Z_{1}, Z n_{1}\right)+E_{\mathrm{TF}}^{\text {atom }}\left(Z_{2}, Z n_{2}\right) .
$$

$n_{1}$ and $n_{2}$ are determined by that the chemical potential for the atoms should be equal (see Sect. 5) and are thus bounded away from 0.

The quantity $\Gamma$ has been studied in [1] where it was proved that $\Gamma$ is smallest in the neutral case. For the neutral case it was proved in [3] that $R^{7} \Gamma$ is increasing in $R$. We recall that from (21) there exists a constant $r_{0}>0$ such that $R>r_{0} Z^{-1 / 3}$ for fermions. Thus with $R=Z^{-1 / 3} r$, we have $r>r_{0}$ and

$$
\Gamma(\underline{Z} ; R ; N) \geq \Gamma(\underline{Z} ; R ; Z)=Z^{7 / 3} \Gamma(\underline{z} ; r ; 1) \geq R^{-7} r_{0}^{7} \Gamma\left(\underline{z} ; r_{0} ; 1\right)=c_{0} R^{-7},
$$

where we have used the scaling property of Thomas-Fermi theory. 
We choose two integers $N_{1}, N_{2}$ with $N_{1}+N_{2}=N$ and such that

$$
\left|N_{k}-n_{k} Z\right| \leq 1 \text { for } k=1,2 \text {. }
$$

Then $N_{1}=Z\left(n_{1}+O\left(Z^{-1}\right)\right)$ and since $E_{\mathrm{TF}}$ is not only an approximate lower bound to the true energy but also an approximate upper bound, we find

$$
E^{\text {atom }}\left(Z_{1}, N_{1}\right) \leq E_{\mathrm{TF}}^{\text {atom }}\left(Z_{1}, n_{1}, Z\right)+c Z_{1}^{7 / 3(1-\varepsilon)} .
$$

We have proved

Proposition 3. If $H(N, \underline{Z}, \underline{\mathbf{R}})$ is the Hamiltonian for a diatomic molecule with $R>$ $r_{0} Z^{-1 / 3}$ (which holds if $H$ has a stable ground state) then there exist $N_{1}, N_{2}$ with $N_{1}+N_{2}=N$ if $N<Z$, or $N_{1}+N_{2}=Z+O\left(Z^{-1}\right)$ if $N \geq Z$, such that

$$
\begin{aligned}
H(N, \underline{Z}) \geq & E^{\text {atom }}\left(Z_{1}, N_{1}\right)+E^{\text {atom }}\left(Z_{2}, N_{2}\right) \\
& +K\left(x_{1} \ldots, x_{N} ; \tilde{\varrho}\right)+c_{0} R^{-7}-C Z^{(7 / 3)(1-\varepsilon)},
\end{aligned}
$$

where

$$
\tilde{\varrho}(x)=\tilde{\varrho}_{N}(x)=\left\{\begin{array}{lll}
\varrho_{\mathrm{TF}}^{\left((1-\delta) \gamma_{p}\right)}(x ; \underline{Z} ; \underline{\mathbf{R}} ; N) & \text { if } & N<Z \\
\varrho_{\mathrm{TF}}^{\left((1-\delta) \gamma_{p}\right)}(x ; \underline{Z} ; \underline{\mathbf{R}} ; Z) & \text { if } & N \geq Z
\end{array},\right.
$$

with $\delta=Z^{-1 / 30}$.

Remark. Above we only described the case $N \leq Z$. The case $N>Z$ is similar. We know from Theorem 1 that $N$ is always of the same order as $Z$.

Remark. Notice that $\tilde{\varrho}_{N}=(1-\delta)^{-3} \varrho^{\gamma_{p}}\left((1-\delta)^{-1} x\right)$.

Since for a stable ground state

$$
0<\Delta E_{b}(N, \underline{Z}) \leq E^{\text {atom }}\left(Z_{1}, N_{1}\right)+E^{\text {atom }}\left(Z_{2}, N_{2}\right)-E(N, \underline{Z}),
$$

we immediately conclude

$$
0<\Delta E_{b}(N, \underline{Z}) \leq C Z^{(7 / 3)(1-\varepsilon)}-\left\{\left\langle\psi\left|K\left(x_{1} \ldots, x_{N} ; \tilde{\varrho}\right)\right| \psi\right\rangle+c_{0} R^{-7}\right\} .
$$

Observing that $K \geq 0$ this proves (7) and (8) of Theorem 2 .

Equation (35) also gives a bound on $K$, this bound will be used to prove (6). We will use $K$ to compare the true quantum mechanical density $\varrho$ with $\varrho$. For atoms a similar technique was employed in [5]. The next step in this comparison is to study the structure of $\tilde{\varrho}$.

\section{The Behavior of the Thomas-Fermi Density for Large Internuclear Distance}

Equation (8) shows that $R$ is much larger than the Thomas-Fermi scale $Z^{-1 / 3}$. The density $\varrho$ will therefore be almost equal to the sum of the densities of two independent atoms. In this section we will control this approximation using some well known monotonicity properties of Thomas-Fermi theory. We will work with the scaled variables $\underline{z}, n, \underline{\mathbf{r}}$, and $\gamma=1$. Corresponding to the density $\varrho_{\mathrm{TF}}$ we define the Thomas-Fermi potential

$$
\varphi_{\mathrm{TF}}(x)=z_{1}\left|x-\mathbf{r}_{1}\right|^{-1}+z_{2}\left|x-\mathbf{r}_{2}\right|^{-1}-\varrho_{\mathrm{TF}} *|x|^{-1}
$$


The ratio $n /\left(z_{1}+z_{2}\right)=n$ and the distance $r$ determines the chemical potential $\mu(r) \geq 0$ for the molecule. We denote by $\varphi_{1}$ and $\varphi_{2}$ the solutions to the atomic TFequations (see [6]) centered at $\mathbf{r}_{1}$ and $\mathbf{r}_{2}$ respectively, and with the same chemical potential $\mu(r)$ as for the molecule. This means that the corresponding electron "numbers" $n_{k}(r), k=1,2$ satisfy (see the review in [6]) $\bar{\mu}\left(n_{k} / z_{k}\right)=z_{k}^{-4 / 3} \mu(r)$. Here $\bar{\mu}(\lambda)$ is the chemical potential for an atom with $z=1$ and $\lambda$ electrons. $\bar{\mu}$ is strictly decreasing and convex for $\lambda<1$ and $(1-\lambda)^{-4 / 3} \bar{\mu}(\lambda)$ is bounded above and below by positive constants for $\lambda$ away from 0 . It is then not hard to see that

$$
0<c<\left|\left(n_{1}(r)-n_{1}(\infty)\right) /\left(n_{2}(r)-n_{2}(\infty)\right)\right|<C,
$$

for large $r$. Notice that we are not claiming that $n_{1}(r)+n_{2}(r)$ will give the total number of electrons $n$. This will only be true as $r \rightarrow \infty$, see Corollary 5 below. Indeed $n_{k}(\infty), k=1,2$ are identical to the $n_{k}$ defined in (32).

The monotonicity property of TF theory (see [6]) gives

$$
\max \left\{\varphi_{1}(x), \varphi_{2}(x)\right\} \leq \varphi(x) \leq \varphi_{1}(x)+\varphi_{2}(x),
$$

where we have omitted the TF-subscript.

Using the TF-equation $\left(\varrho^{2 / 3}=[\varphi-\mu]_{+}\right)$, and the fact that $\varphi_{i}(x) \leq c\left|x-\mathbf{r}_{i}\right|^{-4}$ we immediately conclude the following estimate on the corresponding densities.

\section{Proposition 4.}

$$
0 \leq \varrho(x)-\max \left\{\varrho_{1}(x), \varrho_{2}(x)\right\} \leq C \min \left\{\left|x-\mathbf{r}_{2}\right|^{-2}\left|x-\mathbf{r}_{1}\right|^{-4},\left|x-\mathbf{r}_{1}\right|^{-2}\left|x-\mathbf{r}_{2}\right|^{-4}\right\} .
$$

It is now an easy exercise to prove

Corollary 5. For large $r$,

$$
\left|n-\left(n_{1}(r)+n_{2}(r)\right)\right| \leq C r^{-3} .
$$

Corollary 6. If $r^{\prime} \leq r / 2$ then

$$
\int_{\left|x-\mathbf{r}_{k}\right|<r^{\prime}} \varrho(x) d x=n_{k}(\infty)+O\left(r^{\prime-3}\right) .
$$

Proof. From the proposition,

$$
0 \leq \int_{\left|x-\mathbf{r}_{k}\right|<r^{\prime}} \varrho(x) d x-\int_{\left|x-\mathbf{r}_{k}\right|<r^{\prime}} \max \left\{\varrho_{1}, \varrho_{2}\right\} d x \leq C r^{-4} r^{\prime} \leq C r^{\prime-3} .
$$

But

$$
\int_{\left|x \rightarrow \mathbf{r}_{k}\right|<r^{\prime}} \varrho_{k}(x) d x \leq \int_{\left|x-\mathbf{r}_{k}\right|<r^{\prime}} \max \left\{\varrho_{1}, \varrho_{2}\right\} d x \leq \int_{\left|x-\mathbf{r}_{k}\right|<r^{\prime}} \varrho_{k}(x) d x+C r^{-3}
$$

and

$$
n_{k}(r)=\int \varrho_{k}(x) d x=\int_{\left|x-\mathbf{r}_{k}\right|<r^{\prime}} \varrho_{k}(x) d x+O\left(r^{\prime-3}\right) .
$$

The result now follows from Corollary 5 . 


\section{Estimate on the Electronic Density}

The main technical step in controlling the number of electrons is to prove the following estimate on the 2-point correlation function. A similar estimate was proved for atoms in [12].

Lemma 7. Let $\theta \in C^{\infty}\left(\mathbb{R}^{3}\right)$ with $0 \leq \theta \leq 1,|\nabla \theta|<c_{1} R^{-1}$ and such that $\left|x-\mathbf{R}_{k}\right|>$ $c_{2} R, k=1,2$ on $\operatorname{supp} \theta$, and let $\chi \in C^{\infty}\left(\mathbb{R}^{3} \times \mathbb{R}^{3}\right)$ with $0 \leq \chi$ and $\chi_{x}=\chi(x, \cdot)$ compactly supported. Then

$$
\begin{aligned}
\mid \int & {\left[\varrho^{(2)}(x, y)-\tilde{\varrho}_{N-1}(y) \varrho(x)\right] \theta(x)^{2} \chi(x, y) d x d y \mid } \\
\leq & C \int \varrho(x) \theta(x)^{2} d x\left\{Z^{(7 / 6)(1-\varepsilon)} \sup _{x}\left\|\nabla_{y} \chi_{x}\right\|_{L^{2}\left(\mathbb{R}^{3}\right)}+Z^{1 / 2}\left\|\nabla_{y} \chi\right\|_{L^{\infty}}\right\} \\
& +c Z R^{-1} \sup _{x}\left\|\nabla_{y} \chi\right\|_{L^{2}\left(\mathbb{R}^{3}\right)},
\end{aligned}
$$

where $\varrho_{N-1}$ was defined in (34) and $\varrho, \varrho^{(2)}$ are the true quantum mechanical correlation functions for the stable ground state of $H$.

Proof. For $g_{s}$ as defined in Sect. 4 we have with the choice of $s$ made there,

$$
\left|\chi_{x} * g_{s}^{2}-\chi_{x}\right| \leq s\|\nabla \chi\|_{L^{\infty}} \leq\|\nabla \chi\|_{L^{\infty}} Z^{-1 / 2} .
$$

It is then not difficult to see that

$$
\begin{aligned}
& \left|\int\left[\varrho^{(2)}(x, y)-\tilde{\varrho}(y) \varrho(x)\right] \theta(x)^{2} \chi(x, y) d x d y\right| \\
& \leq C Z^{1 / 2}\|\nabla \chi\|_{L^{\infty}} \int \varrho(x) \theta(x)^{2} d x \\
& \quad+\left\{N \int|\psi(\underline{x})|^{2} \theta\left(x_{1}\right)^{2}\left[\int\left(\sum_{i=2}^{N} g_{s}^{2}\left(y-x_{i}\right)-\tilde{\varrho}(y)\right) \chi\left(x_{1}, y\right) d y\right]^{2} d^{3 N} \underline{x}\right\}^{1 / 2} \\
& \quad \times\left\{\int \varrho \theta^{2} d x\right\}^{1 / 2} .
\end{aligned}
$$

Now using the Fourier transform (denoted by ${ }^{\wedge}$ ) we estimate

$$
\begin{aligned}
& {\left[\int\left(\sum_{i=2}^{N} g_{s}^{2}\left(y-x_{i}\right)-\tilde{\varrho}(y)\right) \chi(x, y) d y\right]^{2}} \\
& \quad \leq \int\left|\hat{\chi}_{x}\right|^{2}|p|^{2} d p \int\left|\left(\sum_{i=2}^{N} g_{s}^{2}\left(\cdot-x_{i}\right)-\tilde{\varrho}\right) \chi(p)\right|^{2}|p|^{-2} d p \\
& \quad=\text { const }\left\|\nabla_{y} \chi_{x}\right\|_{L^{2}\left(\mathbb{R}^{3}\right)}^{2} K_{N-1}\left(x_{2}, \ldots, x_{N} ; \tilde{\varrho}\right),
\end{aligned}
$$

where $K_{N-1}$ was defined in (29). 
Using the IMS-formula and the fact that we are in the ground state we find that

$$
\begin{aligned}
& E(N, \underline{Z}) \int \varrho(x) \theta(x)^{2} d x \\
& \geq \sum_{i=1}^{N}\left\langle\left.\psi\left|\theta\left(x_{i}\right) H(N-1, \underline{Z}, \underline{\mathbf{R}}) \theta\left(x_{i}\right)-\right| \nabla \theta\left(x_{i}\right)\right|^{2}\right. \\
& \quad+\theta\left(x_{i}\right)^{2}\left(\sum_{j, j \neq i}\left|x_{i}-x_{j}\right|^{-1}-Z_{1}\left|x_{i}-\mathbf{R}_{1}\right|^{-1}-Z_{2}\left|x_{i}-\mathbf{R}_{2}\right|^{-1}\right)|\psi\rangle
\end{aligned}
$$

where in the $i^{\text {th }}$ term we have neglected the positive $-\Delta_{i}$ term. Since the ground state is stable we know that actually

$$
E(N, Z)<\min _{N_{1}^{\prime}+N_{2}^{\prime} \leq N}\left(E^{\text {atom }}\left(Z_{1}, N_{1}^{\prime}\right)+E^{\text {atom }}\left(Z_{2}, N_{2}^{\prime}\right)\right) .
$$

From Proposition 3 (used for $N-1$ ) we can thus estimate the right-hand side of (38) below by

$$
\begin{aligned}
E(N, \underline{Z}) \int \varrho(x) \theta(x)^{2} d x & +N \int|\psi|^{2} K_{N-1} \theta\left(x_{1}\right)^{2} d^{3 N} \underline{x} \\
& -C\left(Z^{7(1-\varepsilon) / 3}+Z R^{-1}\right) \int \varrho(x) \theta(x)^{2} d x-c N R^{-2} .
\end{aligned}
$$

In the last inequality we have thrown away the term coming from the repulsion between the electrons and used the support property for $\theta$. Thus since $R \geq$ $C_{3} Z^{-(1 / 3)(1-\varepsilon)}$ we get

$$
N \int|\psi|^{2} K_{N-1}\left(x_{2}, \ldots, x_{N}\right) \theta\left(x_{1}\right)^{2} d^{3 N} \underline{x} \leq C Z^{7(1-\varepsilon) / 3} \int \varrho(x) \theta(x)^{2} d x+c N R^{-2} .
$$

Putting everything together and using $\int \varrho \theta^{2} \leq N \leq$ const $Z$ gives (37).

A simple modification of the above proof gives

Corollary 8. For all $\chi \in C_{0}^{\infty}\left(\mathbb{R}^{3}\right)$,

$$
\left|\int\left[\varrho(x)-\tilde{\varrho}_{N-1}(x)\right] \chi(x) d x\right| \leq Z^{(7 / 6)(1-\varepsilon)}\|\nabla \chi\|_{L^{2}}+Z^{1 / 2}\|\nabla \chi\|_{L^{\infty}} .
$$

\section{Lower Bound on $N$}

In this section we prove the lower bound on the number of electrons. We will assume that $N<Z$. We begin by defining new localizing functions for fermions. Choose $\theta_{1}, \theta_{2} \in C_{0}^{\infty}\left(\mathbb{R}^{3}\right)$ and $\theta_{0} \in C^{\infty}\left(\mathbb{R}^{3}\right)$ such that they satisfy the following conditions.

1. $0 \leq \theta_{0}, \theta_{1}, \theta_{2} \leq 1$.

2. For $k=1,2, \theta_{k}$ depends only on $\left|x-\mathbf{R}_{k}\right|$,

$$
\operatorname{supp} \theta_{k} \subset\left\{x|| x-\mathbf{R}_{k} \mid<\frac{1}{4} R\right\}
$$

and $\theta_{k}=1$ for $\left|x-\mathbf{R}_{k}\right|<(1 / 5) R$. 
3. $\left|\nabla \theta_{0}\right|,\left|\nabla \theta_{1}\right|,\left|\nabla \theta_{2}\right| \leq C R^{-1}$.

4. $\theta_{0}(x)^{2}+\theta_{1}(x)^{2}+\theta_{2}(x)^{2}=1$.

For $k=1$ or $2, \theta_{k}$ localizes close to the nucleus at $\mathbf{R}_{k}$. As our full localizing functions we then choose

$$
\chi_{1}(x)=\left\{\theta_{1}(x)^{2}+\alpha_{1} \theta_{0}(x)^{2}\right\}^{1 / 2} \text { and } \chi_{2}(x)=\left\{\theta_{2}(x)^{2}+\alpha_{2} \theta_{0}(x)^{2}\right\}^{1 / 2},
$$

where $0 \leq \alpha_{1}, \alpha_{2} \leq 1$ and $\alpha_{1}+\alpha_{2}=1$, we are not going to make any explicit choice for $\alpha_{1}, \alpha_{2}$ here since it is not important for the general argument. We will keep them in the following derivation so as to simplify the identification of different terms. Then

$$
\chi_{1}(x)^{2}+\chi_{2}(x)^{2}=1
$$

The point is that we localize the core electrons, i.e., electrons in $\operatorname{supp} \theta_{1} \cup \operatorname{supp} \theta_{2}$, differently from the outer electrons, i.e., electrons in $\operatorname{supp} \theta_{0}$. An important step in our analysis is to prove that almost all electrons belong to one of the two cores.

From (16) we find

$$
\begin{aligned}
-\Delta E_{b} \geq & -c N R^{-2}-\sum_{i=1}^{N}\langle\psi|\left(Z_{1}\left|x_{i}-\mathbf{R}_{1}\right|^{-1}-\sum_{j \neq i} \theta_{1}\left(x_{j}\right)^{2}\left|x_{i}-x_{j}\right|^{-1}\right) \alpha_{2} \theta_{0}\left(x_{i}\right)^{2} \\
& +\left(Z_{2}\left|x_{i}-\mathbf{R}_{2}\right|^{-1}-\sum_{j \neq i} \theta_{2}\left(x_{j}\right)^{2}\left|x_{i}-x_{j}\right|^{-1}\right) \alpha_{1} \theta_{0}\left(x_{i}\right)^{2}|\psi\rangle \\
& -\sum_{i=1}^{N}\left\langle\psi\left|Z_{1}\right| x_{i}-\left.\mathbf{R}_{1}\right|^{-1} \theta_{2}\left(x_{i}\right)^{2}+Z_{2}\left|x_{i}-\mathbf{R}_{2}\right|^{-1} \theta_{1}\left(x_{i}\right)^{2} \mid \psi\right\rangle \\
& +\sum_{1 \leq i \leq j \leq N}\left\langle\psi\left|\left(\theta_{1}\left(x_{i}\right)^{2} \theta_{2}\left(x_{j}\right)^{2}+\theta_{2}\left(x_{i}\right)^{2} \theta_{1}\left(x_{j}\right)^{2}\right)\right| x_{i}-\left.x_{j}\right|^{-1} \mid \psi\right\rangle+Z_{1} Z_{2} / R \\
= & -c N R^{-2}-A_{1}-A_{2}+B .
\end{aligned}
$$

Where we have defined the "attraction" between the $k^{\text {th }}$ screened nucleus, $k=1,2$ and the outer electrons in the other cluster $\bar{k}$,

$$
\begin{aligned}
A_{k}= & Z_{k} \int \varrho(x) \alpha_{\bar{k}} \theta_{0}(x)^{2}\left|x-\mathbf{R}_{k}\right|^{-1} d x \\
& -\int \varrho^{(2)}(x, y) \theta_{k}(y)^{2} \alpha_{\bar{k}} \theta_{0}(x)^{2}|x-y|^{-1} d x d y,
\end{aligned}
$$

and the "repulsion" between the screened nuclei

$$
\begin{aligned}
B= & Z_{1} Z_{2} / R+\int \varrho^{(2)}(x, y)|x-y|^{-1} \theta_{1}(x)^{2} \theta_{2}(y)^{2} d x d y \\
& -Z_{1} \int \varrho(x) \theta_{2}(x)^{2}\left|x-\mathbf{R}_{1}\right|^{-1} d x-Z_{2} \int \varrho(x) \theta_{1}(x)^{2}\left|x-\mathbf{R}_{2}\right|^{-1} d x .
\end{aligned}
$$

The reason why we put attraction and repulsion in quotation marks is that we do not know a priori that $A_{k}, B$ are positive.

Notice that in contrast with Sect. 3 we have here made use of part of the electron-electron repulsion term in (16). 
We will now estimate $A_{k}, B$ using the results from the previous sections.

Let $\tilde{R}=C_{3} Z^{-(1 / 3)(1-\varepsilon)}$ then from (8) $\tilde{R} \leq R$. Choose $\tilde{\theta}_{k} \in C_{0}^{\infty}\left(\mathbb{R}^{3}\right)$ with the same properties as $\theta_{k}$ except that

$$
\tilde{\theta}_{k}(y)= \begin{cases}1 & \text { if } \quad\left|y-\mathbf{R}_{k}\right|<\tilde{R} / 7 \\ 0 & \text { if } \quad\left|y-\mathbf{R}_{k}\right|>\tilde{R} / 6\end{cases}
$$

and $\left\|\nabla \tilde{\theta}_{k}\right\|_{\infty} \leq c \tilde{R}^{-1}$. Then $\tilde{\theta}_{k} \leq \theta_{k}$. We will estimate $A_{k}$ by Lemma 7 with $\theta=\theta_{0}$ and

$$
\chi(x, y)=\alpha_{\bar{k}}|x-y|^{-1} \tilde{\theta}_{k}(y)^{2} .
$$

Notice that if $\theta(x)^{2} \chi(x, y) \neq 0$, then $|x-y|>c R$ and thus for these $x$

$$
\left\|\nabla_{y} \chi_{x}\right\|_{L^{2}\left(\mathbb{R}^{3}\right)} \leq c \tilde{R}^{1 / 2} R^{-1} \text { and }\left|\nabla_{y} \chi_{x}\right| \leq c \tilde{R}^{-1} R^{-1} .
$$

From Lemma 7 we can now conclude

$$
\begin{aligned}
A_{k} \leq & Z_{k} \int \varrho(x) \alpha_{\bar{k}} \theta_{0}(x)^{2}\left|x-\mathbf{R}_{k}\right|^{-1} d x \\
& -\int \varrho^{(2)}(x, y) \alpha_{\bar{k}} \theta_{0}(x)^{2}|x-y|^{-1} \tilde{\theta}_{k}(y)^{2} d x d y \\
\leq & Z_{k} \int \varrho(x) \alpha_{\bar{k}} \theta_{0}(x)^{2}\left|x-\mathbf{R}_{k}\right|^{-1} d x \\
& -\int \varrho(x) \tilde{\varrho}_{N-1}(y) \alpha_{\bar{k}} \theta_{0}(x)^{2}|x-y|^{-1} \tilde{\theta}_{k}(y)^{2} d x d y \\
& +c Z^{1-\varepsilon} R^{-1} \int \varrho(x) \theta_{0}(x)^{2} d x+c Z^{(5+\varepsilon) / 6} R^{-2} .
\end{aligned}
$$

If $N_{1}$ and $N_{2}$ satisfy $N_{1}+N_{2}=N$ and $N_{k} / Z-n_{k}=O\left(Z^{-1}\right)$ then using Corollary 6 in Sect. 5 and the definition of $\tilde{R}$ we find

$$
\int \tilde{\varrho}_{N-1}(y) \tilde{\theta}_{k}(y)^{2} d y=N_{k}+(1-\delta)^{3} O\left(\tilde{R}^{-3}\right)=N_{k}+O\left(Z^{1-\varepsilon}\right),
$$

and in (42) the order of the error term will not be increased by replacing $\tilde{\varrho}_{N-1}$ by the spherically symmetric TF density for an atom with $N_{k}$ electrons centered at $\mathbf{R}_{k}$, i.e.,

$$
\begin{aligned}
A_{k} \leq & \left(Z_{k}-N_{k}+c Z^{1-\varepsilon}\right) \alpha_{\bar{k}} \int \varrho(x) \theta_{0}(x)^{2}\left|x-\mathbf{R}_{k}\right|^{-1} d x \\
& +c Z^{1-\varepsilon} R^{-1} \int \varrho(x) \theta_{0}(x)^{2} d x+c Z^{(5+\varepsilon) / 6} R^{-2} .
\end{aligned}
$$

Now by Corollary 8

$$
\int \varrho(x) \theta_{0}(x)^{2} d x=N-\int \varrho(x)\left(\theta_{1}(x)^{2}+\theta_{2}(x)^{2}\right) d x=O\left(Z^{1-\varepsilon}\right) .
$$

Thus again using (8) we finally arrive at

$$
A_{1}+A_{2} \leq C\left(\alpha_{2}\left(Z_{1}-N_{1}\right)+\alpha_{1}\left(Z_{2}-N_{2}\right)+c Z^{1-\varepsilon}\right) Z^{1-\varepsilon} R^{-1} .
$$

Likewise for $B$

$$
B \geq C\left(Z_{1}-N_{1}\right)\left(Z_{2}-N_{2}\right) R^{-1}-c Z^{2-\varepsilon} R^{-1} .
$$


By changing the choice of $\varepsilon,(\varepsilon \rightarrow \varepsilon / 2)$ we can still write the error term above as $c Z^{2(1-\varepsilon)}$.

Inserting these two bounds into (39) and using $\Delta E_{b}>0$ gives

$$
\left(Z_{1}-N_{1}\right)\left(Z_{2}-N_{2}\right)-c Z^{1-\varepsilon}\left(\left(Z_{1}-N_{1}\right)+\left(Z_{2}-N_{2}\right)\right)-c Z^{2(1-\varepsilon)} \leq c Z^{(4 / 3-\varepsilon / 3)},
$$

or

$$
\left(1-N_{1} / Z_{1}\right)\left(1-N_{2} / Z_{2}\right)-c Z^{-\varepsilon}\left(1-N_{1} / Z_{1}\right)-c Z^{-\varepsilon}\left(1-N_{2} / Z_{2}\right) \leq c Z^{-2 \varepsilon},
$$

the constant $c$ depends only on $z_{1}$ and $z_{2}$.

From $\bar{\mu} \approx c(1-\lambda)^{4 / 3}$ for $\lambda \approx 1$ (see Sect. 5) we get that $\left|\left(1-N_{1} / Z_{1}\right) /\left(1-N_{2} / Z_{2}\right)\right|$ is bounded above and below and hence $1-N_{k} / Z_{k} \leq c Z^{-\varepsilon}$. This is the lower bound on $N$ given in Theorem 2 .

\section{Upper Bound on $N$}

The upper bound that we prove in this section is similar to excess charge bounds for atoms (see [5 or 12]). From our comparison with Thomas-Fermi theory we know that the nuclei are almost screened. By copying the proof in [7] we will conclude that there cannot be too many electrons outside the screened nuclei.

Define

$$
\varphi(x)=z_{1}\left|x-\mathbf{R}_{1}\right|^{-1}+z_{2}\left|x-\mathbf{R}_{2}\right|^{-1} .
$$

With $\theta_{0}$ as in the previous section we compute

$$
\begin{aligned}
E(N, \underline{Z}) \int \varrho(x) \theta_{0}(x)^{2} \varphi(x)^{-1} d x \\
=\sum_{i=1}^{N}\left\langle\psi\left|\theta_{0}\left(x_{i}\right)^{2} \varphi\left(x_{i}\right)^{-1} H(N, \underline{Z})\right| \psi\right\rangle \\
\quad \geq \sum_{i=1}^{N}\left(E(N-1, \underline{Z})\left\langle\psi\left|\theta_{0}\left(x_{i}\right)^{2} \varphi\left(x_{i}\right)^{-1}\right| \psi\right\rangle-\left\langle\psi\left|\left(\nabla_{i}\left(\theta_{0} \varphi^{-1 / 2}\right)\right)^{2}\right| \psi\right\rangle\right) \\
\quad+\sum_{i=1}^{N}\left\langle\psi\left|\theta_{0}\left(x_{i}\right)^{2} \varphi\left(x_{i}\right)^{-1}\left(-Z_{1}\left|x_{i}-\mathbf{R}_{1}\right|-Z_{2}\left|x_{i}-\mathbf{R}_{2}\right|+\sum_{j, j \neq i}\left|x_{i}-x_{j}\right|^{-1}\right)\right| \psi\right\rangle,
\end{aligned}
$$

or since $\left|\nabla\left(\theta_{0} \varphi^{-1 / 2}\right)\right|<C R^{-1 / 2}$,

$$
\begin{aligned}
0 \geq & -Z \int \varrho(x) \theta_{0}(x)^{2} d x+\int \varrho^{(2)}(x, y) \theta_{0}(x)^{2} \varphi(x)^{-1}\left[1-\theta_{0}(y)^{2}\right]|x-y|^{-1} d x d y \\
& +\frac{1}{2} \int \varrho^{(2)}(x, y) \theta_{0}(x)^{2}\left(\varphi(x)^{-1}+\varphi(y)^{-1}\right) \theta_{0}(y)^{2}|x-y|^{-1} d x d y-c N R^{-1}
\end{aligned}
$$

We estimate the different terms independently using Lemma 7. Since we can assume $N \geq Z+1$ we have this time that $\varrho_{N-1}=\varrho_{Z}$ is the neutral TF density. If we let $\tilde{\theta}_{k}$ be defined as in the previous section we will use Lemma 7 with $\theta(x)=\theta_{0}(x)$ and

$$
\chi(x, y)=2\left[\tilde{\theta}_{1}(y)^{2}+\tilde{\theta}_{2}(y)^{2}\right] \varphi(x)^{-1}|x-y|^{-1} .
$$


Then for $x \in \operatorname{supp} \theta_{0}$ we have

$$
\left|\nabla_{y} \chi_{x}\right| \leq C \tilde{R}^{-1} \text { and }\left\|\nabla_{y} \chi_{x}\right\|_{L^{2}\left(\mathbb{R}^{3}\right)} \leq C \tilde{R}^{1 / 2} .
$$

First recall that

$$
\begin{aligned}
& \int \varrho^{(2)}(x, y) \theta_{0}(x)^{2}\left[1-\theta_{0}(y)^{2}\right] \varphi(x)^{-1}|x-y|^{-1} d x d y \\
& \quad \geq \int \varrho^{(2)}(x, y) \theta_{0}(x)^{2}\left[\tilde{\theta}_{1}(y)^{2}+\tilde{\theta}_{2}(y)^{2}\right] \varphi(x)^{-1}|x-y|^{-1} d x d y,
\end{aligned}
$$

and thus from Lemma 7

$$
\begin{aligned}
& \int \varrho^{(2)}(x, y) \theta_{0}(x)^{2}\left[1-\theta_{0}(y)^{2}\right] \varphi(x)^{-1}|x-y|^{-1} d x d y \\
& \geq \int \varrho(x) \varrho(y) \theta_{0}(x)^{2}\left[\tilde{\theta}_{1}(y)^{2}+\tilde{\theta}_{2}(y)^{2}\right] \varphi(x)^{-1}|x-y|^{-1} d x d y \\
& \quad-c\left[\tilde{R}^{1 / 2} Z^{(7 / 6)(1-\varepsilon)}+Z^{1 / 2} \tilde{R}^{-1}\right] \int \varrho(x) \theta_{0}(x)^{2} d x-c Z R^{-1} \tilde{R}^{1 / 2} \\
& \quad \geq\left(Z-c Z^{(1-\varepsilon)}\right) \int \varrho(x) \theta_{0}(x)^{2} d x-c Z^{(7 / 6-\varepsilon / 6)} .
\end{aligned}
$$

As in (43) we find from Corollary 8 ,

$$
N-Z+C_{-} Z^{(1-\varepsilon)} \leq \int \varrho(x) \theta_{0}(x)^{2} d x \leq N-Z+C_{+} Z^{(1-\varepsilon)} .
$$

We can therfore conclude that

$$
\int \varrho^{(2)}(x, y) \theta_{0}(x)^{2}\left[1-\theta_{0}(y)^{2}\right] \varphi(x)^{-1}|x-y|^{-1} d x d y \geq\left(Z-C Z^{(1-\varepsilon)}\right) \varrho(x) \theta_{0}(x)^{2} d x .
$$

In estimating the next term in (44) we follow the argument in [7] and use the triangle inequality to conclude

$$
\varphi(x)^{-1}+\varphi(y)^{-1} \geq \sum_{k=1}^{2} z_{k}\left[\varphi(x)\left|x-\mathbf{R}_{k}\right|\right]^{-1}\left[\varphi(y)\left|y-\mathbf{R}_{k}\right|\right]^{-1}|x-y|
$$

We now appeal to the general inequality

$$
\int \varrho^{(2)}(x, y) f(x) f(y) d x d y \geq\left(\int \varrho(x) f(x) d x\right)^{2}-\int \varrho(x) f(x)^{2} d x,
$$

which follows from $\left\langle F^{2}\right\rangle-\langle F\rangle^{2} \geq 0$. We arrive at

$$
\begin{aligned}
\int & \varrho^{(2)}(x, y) \theta_{0}(x)^{2}\left(\varphi(x)^{-1}+\varphi(y)^{-1}\right) \theta_{0}(y)^{2}|x-y|^{-1} d x d y \\
\geq & \sum_{k=1}^{2} z_{k}\left\{\left(\int \varrho(x) \theta_{0}(x)^{2}\left[\varphi(x)\left|x-\mathbf{R}_{k}\right|\right]^{-1} d x\right)^{2}\right. \\
& \left.-\int \varrho(x) \theta_{0}(x)^{4}\left[\varphi(x)\left|x-\mathbf{R}_{k}\right|\right]^{-2}\right\} d x \\
\geq & C_{1}\left(\int \varrho(x) \theta_{0}(x)^{2} d x\right)^{2} C_{2} \int \varrho(x) \theta_{0}(x)^{2} d x,
\end{aligned}
$$


where we have used that $\theta_{0} \leq 1$ and that on $\operatorname{supp} \theta_{0}$,

$$
c_{-} \leq \varphi(x)\left|x-\mathbf{R}_{k}\right| \leq c_{+} .
$$

Inserting all this into (44) and again using (8) on $R$ we conclude

$$
\int \varrho(x) \theta_{0}(x)^{2} d x \leq C Z^{(1-\varepsilon)},
$$

and hence from (45) the desired result

$$
N \leq Z+C Z^{(1-\varepsilon)} .
$$

This completes the proof of the asymptotic neutrality.

Ac nowledgements. I would like to thank Elliott Lieb and Mary Beth Ruskai for helpful discussions and comments.

\section{References}

1. Benguria, R.: Dependence of the Thomas-Fermi energy on the nuclear coordinates. Commun. Math. Phys. 81, 419-428 (1981)

2. Benguria, R., Lieb, E:H.: Proof of stability of highly negative ions in the absence of the Pauli principle. Phys. Rev. Lett. 50, 1771-1774 (1983)

3. Brezis, H., Lieb, E:H.: Long range potentials in Thomas-Fermi theory. Commun. Math. Phys. 65, 231-246 (1979)

4. Cycon, H.L., Froese, R.G., Kirsch, W., Simon, B.: Schrödinger Operators. Berlin, Heidelberg, New York: Springer 1987

5. Fefferman, C.L., Seco, L.A.: Asymptotic neutrality of large ions. Commun. Math. Phys. 1989 (in press)

6. Lieb, E.H.: Thomas-Fermi and related theories of atoms and molecules. Rev. Mod. Phys. 53, 603-641 (1981)

7. Lieb, E.H.: Bound on the maximum negative ionization of atoms and molecules. Phys. Rev. A 29, 3018-3028 (1984)

8. Lieb, E.H., Sigal, I.M., Simon, B., Thirring, W.: Asymptotic neutrality of large- $Z$ ions. Commun. Math. Phys. 116, 635-644 (1988)

9. Lieb, E.H., Thirring, W.E.: Universal nature of the van der Waals forces for Coulomb systems. Phys. Rev. A 34, 40-46 (1986)

10. Ruskai, M.B.: Limit on the excess negative charge of a dynamic diatomic molecule. Preprint, 1989

11. Ruskai, M.B.: Limits on stability of positive molecular ions. Lett. Math. Phys. 18, 121-132 (1989)

12. Seco, L.A., Sigal, I.M., Solovej, J.P.: Bound on the ionization energy of large atoms. Commun. Math. Phys. (to appear)

13. Thirring, W.E.: A course in mathematical physics, vol. III. Berlin, Heidelberg, New York: Springer 1981

Communicated by B. Simon

Received August 30, 1989; in revised form November 29, 1989 